This is a postprint version of the following published document:

de la Oliva, A., Li, X., Costa-Pérez, X., Bernardos, C.J., Bertin, P., Iovanna, P., Deiss, T., Mangues, J., Mourad, A., Casetti, C., González, J.E., Azcorra, A. (2018). 5GTRANSFORMER: Slicing and Orchestrating Transport Networks for Industry Verticals. IEEE Communications Magazine, 56(8), pp. 78-84.

DOI:10.1109/MCOM.2018.1700990

(C)2018 IEEE. Personal use of this material is permitted. Permission from IEEE must be obtained for all other uses, in any current or future media, including reprinting/republishing this material for advertising or promotional purposes, creating new collective works, for resale or redistribution to servers or lists, or reuse of any copyrighted component of this work in other works. 


\title{
5G-TRANSFORMER: Slicing and Orchestrating Transport Networks for Industry Verticals
}

\author{
Antonio de la Oliva, Xi Li, Xavier Costa-Pérez, Carlos J. Bernardos, Philippe Bertin, Paola lovanna, Thomas Deiss, Josep Mangues, Alain Mourad, \\ Claudio Casetti, Jose Enrique Gonzalez, and Arturo Azcorra
}

The authors dive into the design of the next generation mobile transport networks to simultaneously support the needs of various vertical industries with a diverse range of networking and computing requirements. Network slicing has emerged as the most promising approach to address this challenge by enabling per-slice management of virtualized resources. They aim to bring the network slicing paradigm into mobile transport networks by provisioning and managing slices tailored to the needs of different vertical industries.

\footnotetext{
1 Core Analysis, Mobile Edge Computing 2016, Market report, April 2016.

2 http://www.5g-transformer. eu/
}

\begin{abstract}
This article dives into the design of the next generation mobile transport networks to simultaneously support the needs of various vertical industries with diverse range of networking and computing requirements. Network slicing has emerged as the most promising approach to address this challenge by enabling per-slice management of virtualized resources. We aim to bring the network slicing paradigm into mobile transport networks by provisioning and managing slices tailored to the needs of different vertical industries. Our technical approach is twofold: (i) enabling vertical industries to meet their service requirements within customized slices; and (ii) aggregating and federating transport networking and computing fabric, from the edge up to the core and cloud, to create and manage slices throughout a federated virtualized infrastructure. The main focus of the article is on major technical highlights of vertical-oriented slicing mechanisms for $5 \mathrm{G}$ mobile networks.
\end{abstract}

\section{INTRODUCTION AND MOTIVATION}

Research and standardization of the upcoming fifth generation (5G) systems have been quite hot areas recently, noticeably in research and industry forums and standards development organizations (SDOs).

In this context, three technologies have emerged as key $5 \mathrm{G}$ pillars:

- Network functions virtualization (NFV) $[1,2]$

- Slicing [3]

- Multi-access (mobile) edge computing (MEC) [4]

In NFV, the network functions are virtualized by properly instantiating, connecting, and combining them over the underlying substrate networks. In slicing, the infrastructure sharing between different tenants highly decreases the operating expediture (OPEX) of the network. MEC also drives OPEX reduction by handling the traffic locally and hence keeping it away from the core network, but its primary purpose is to enable low-latency services. Network softwarization, virtualization, and automation, supported by such commodity hard- ware, will significantly contribute to reduce both capital expenditure (CAPEX) and OPEX between 40 and 50 percent. ${ }^{1}$

Leveraging these technologies, the 5G-TRANSFORMER project ${ }^{2}$ set focus on evolving the mobile transport network toward a software defined networking (SDN)/NFV/MEC-based 5G mobile transport and computing platform (MTP). NFV is gaining incredible momentum among mobile operators as one of the significant solutions to optimize the resource allocation and system scalability in 5G networks. In 5G-TRANSFORMER, each network slice may span across several data centers that provide the virtual processing resources, where network configurations/ adaptations and data forwarding are managed by SDN controllers. Orchestration is therefore a key enabler in 5G-TRANSFORMER to support slicing for different verticals, efficient load distribution, and arbitration among the network slices.

One major challenge in slicing is exposing the capabilities of the network including topologies and resources via proper abstraction to the orchestration layer. 5G-TRANSFORMER will design new models, interfaces, and optimization algorithms to achieve efficient orchestration, interoperability, and integration between different $5 \mathrm{G}$ network sites. The MTP inherits the transport infrastructure of the phase 1 project 5G-Crosshaul [5], defining an integrated network that can transport backhaul and fronthaul over the same transport substrate. In 5G-TRANSFORMER, this network will be extended to better support slicing and MEC, leveraging previous works on end-toend slicing, such as $[6,7]$. In addition, 5G-TRANSFORMER will also include federation of resources from multiple domains, building on top of the research performed on the phase 1 project 5GEx [8], enabling the creation of end-to-end networks consisting of disjoint resources.

The rest of this article is structured as follows. We present the high-level architecture of the system while presenting the main research challenges that must be tackled for the 5G-TRANSFORMER concept to become a reality. Finally, we conclude this work summarizing the key innovations brought by 5G-TRANSFORMER. 


\begin{tabular}{|c|c|}
\hline Vertical description and use cases & Vertical requirements \\
\hline $\begin{array}{l}\text { Automotive: } \\
\text { - Advanced driver assistance systems (ADASs) enabling autonomous driving. } \\
\text { - Onboard systems and smart, interconnected networks for vehicle-to- } \\
\text { everything (V2X). }\end{array}$ & $\begin{array}{l}\text { - Cross-domain network slice for seamless V2X communications. } \\
\text { - Configurable network slice to serve vertical requirements (e.g., reduce delay, prioritize } \\
\text { certain traffic). }\end{array}$ \\
\hline $\begin{array}{l}\text { eHealth: } \\
\text { - Virtualized private network with low-latency coordination mechanisms for } \\
\text { emergency services. } \\
\text { - Medical alerts from wearables for emergency detection and healthcare } \\
\text { coordination. }\end{array}$ & $\begin{array}{l}\text { - On-demand instantiation of slices with dynamic characteristics. } \\
\text { - Integration of slices in the already deployed system, especially with emergency response } \\
\text { call centers. } \\
\text { - Strict priority of traffic and location services. } \\
\text { - Deployment of third-party services over MEC. }\end{array}$ \\
\hline $\begin{array}{l}\text { Media and Entertainment: } \\
\text { - Higher data rates, number of simultaneous users connected, better quality of } \\
\text { experience, etc. } \\
\text { - Immersive sports experience: smart stadiums, AR/VR, } 360^{\circ} \text { streaming. }\end{array}$ & $\begin{array}{l}\text { - Dynamic creation of slices with MEC extended services, such as content distribution networks } \\
\text { (CDNs), allowing isolation of traffic. } \\
\text { - Dynamic deployment of on-the-fly services for geographical areas. } \\
\text { - Strict prioritization of traffic and location services. } \\
\text { - Use of resources from different providers at different venues. }\end{array}$ \\
\hline
\end{tabular}

Table 1. 5G-TRANSFORMER verticals and associated requirements.

\section{THE 5G-TRANSFORMER CONCEPT}

A key novelty of $5 \mathrm{G}$ will be the creation of tailor-made infrastructure to meet vertical industries' requirements. The $5 \mathrm{G}$-TRANSFORMER concept is driven by the automotive, eHealth, and media and entertainment vertical industries. Table 1 introduces these verticals and their associated requirements for $5 \mathrm{G}$ networks.

A 5G-TRANSFORMER slice is defined as a dedicated logical infrastructure provided to verticals to support their services and meeting their specific requirements. It is composed of a set of virtual network functions (VNFs) and/or virtual applications (VAs) and their required (virtual or physical) resources (including networking, computing, and storage). A 5G-TRANSFORMER slice can span some or all domains of the network: software modules including VNFs/VAs running on cloud nodes and/or vertical domains, specific configurations of the 5G transport and core network supporting flexible location of network functions, a dedicated radio configuration, or even configuration of the end devices and/or the applications of vertical users or third-party entities.

The behavior of the network slice is realized via network slice instance(s). The allocation of a 5G-TRANSFORMER slice instance involves:

- The placement of functions constrained by the status of the mobile transport network, the instantiation of VNF/VAs and logical links interconnecting both the VNF/VAs and existing physical systems according to a template and a descriptor

- The partitioning and reservation of resources - either shared or dedicated, physical or virtual - to deploy such VNF/VAs and to provide the required connectivity

- The configuration of the underlying physical infrastructure to meet the requirements and the service level agreements (SLAs) associated to the slice

- The enabling of a set of interfaces to allow the vertical actor to monitor and operate the slice and integrate it with its own operation and business support systems (OSS/BSS)

Figure 1 illustrates the 5G-TRANSFORMER concept. It builds on three main modules (from top to bottom as depicted on the right side of the figure), namely:
- Vertical slicer (VS)

- Service orchestrator (SO)

- MTP

These three modules jointly allow any vertical industry to obtain an end-to-end 5G-TRANSFORMER slice tailored to its needs.

The vertical slicer (VS) is a common entry point for all vertical industries into the 5G-TRANSFORMER system (note that each administrative domain has one VS). It coordinates and arbitrates vertical slice requests for the use of networking and computing resources. Slices are requested at the VS through a newly defined interface using templates (called blueprints) with simple interconnection models, thus relieving the vertical industry from specifying its slice details. The VS is therefore in charge of mapping the high-level requirements and placement constraints of the slice template into a set of one or more VNF/VA graphs and service function chains (SFCs).

The service orchestrator (SO) is the main decision point of the system. It manages the allocation and monitoring of all virtual resources to all slices. Depending on the slice requirements and network context, the SO may interact with other SOs belonging to other administrative authority domains to take decisions on the end-to-end service (de)composition of virtual resources and their most suitable execution environment. This can be a single or multiple administrative domains depending on resources availability and characteristics.

Finally, the MTP manages the underlying physical mobile transport network and computing infrastructure. It evolves the $5 \mathrm{G}-\mathrm{Crosshaul}$ solution to integrate $M E C$ resources from multiple domains and provide support for the 5G-TRANSFORMER concept of slicing. It enforces slice requirements coming from the SO and provides physical infrastructure monitoring and analytics services.

Figure 2 shows the high-level workflow for instantiation of a vertical service through these modules. During service onboarding, the VS defines a set of vertical services in a vertical service catalog offered to the vertical tenants through service advertisement. To request a vertical service, the tenant sends a service request to the VS, which includes the selection of one or multiple services from the provided catalog, including a generic high-level service description. Then the VS translates the high-level service requirements to a service graph, which can be understood as an NFV 


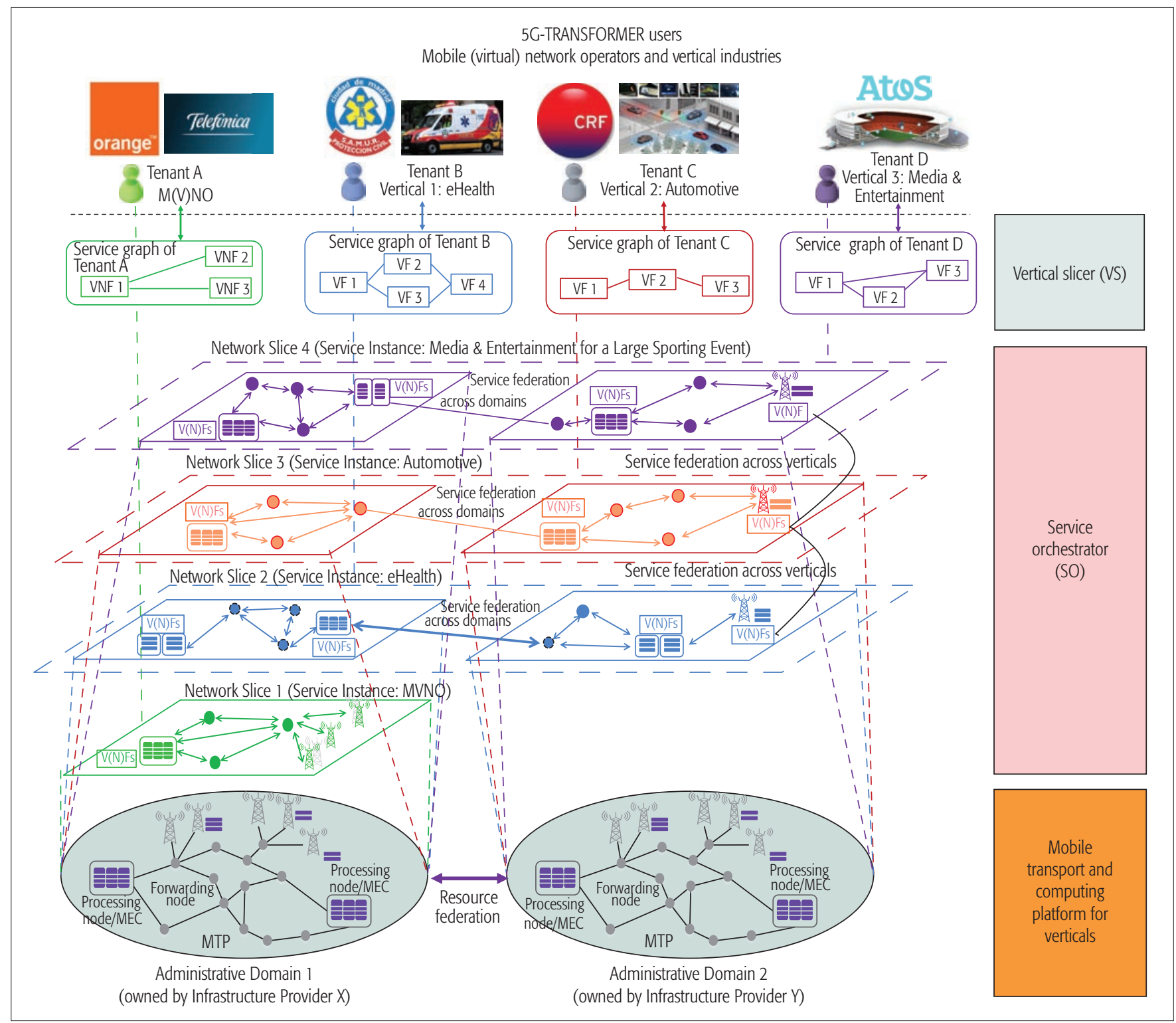

Figure 1. 5G-TRANSFORMER concept.

network service forwarding graph. It can be described by a network service descriptor (NSD) with specified deployment flavors, including the composition of a set of VNFs/VAs chained with each other to build a nested service (i.e., SFCs) and the resource requirements. To request the instantiation of a service, the VS sends the service instance instantiation request to the SO, including the requested service graph. Then the SO maps the service graph to an MTP network slice by means of orchestration of virtual resources to this slice. This is based on the abstraction provided by the local and federated MTPs (whenever federation is needed). The orchestration decision for an MTP slice consists of the placement of VNFs over a virtual network as well as deciding the resources to be allocated. The SO will then request the MTP to instantiate the MTP network slice instance. The MTP is responsible for the actual allocation/instantiation/ control/configuration of virtual resources (including networking, computing, and storage resources) over the underlying physical infrastructure.

The interworking of these modules is presented in a simplified system view in Fig. 3. A key challenge is to define the interfaces and abstractions (adequate level of details) at different levels in the system. To do so, 5G-TRANSFORMER defines four different service access points (SAPS): VS-SAP, SO-SAP, SOSO-SAP, and MTP-SAP.

The VS-SAP interfaces between each vertical slice (described in its slice template) and the VS, which will do the arbitration between the different vertical slices, and map each slice description into VNF graphs and SFCs.

The VS module then interfaces through SO-SAP with the service orchestrator (SO) module. The SO-SAP provides the requirements of each slice in terms of resource allocation and monitoring. Note that for non-vertical slices (e.g., the MVNO slice), the slice interfaces directly through the SO-SAP with the SO (i.e., it does not go through the VS module).

The SO module manages the allocation and monitoring of all resources to all slices. It provides an end-point for federation of resources from multiple providers and multiple domains, including its own domain. The SO initially interacts with the VS to get the resource allocation and monitoring require- 


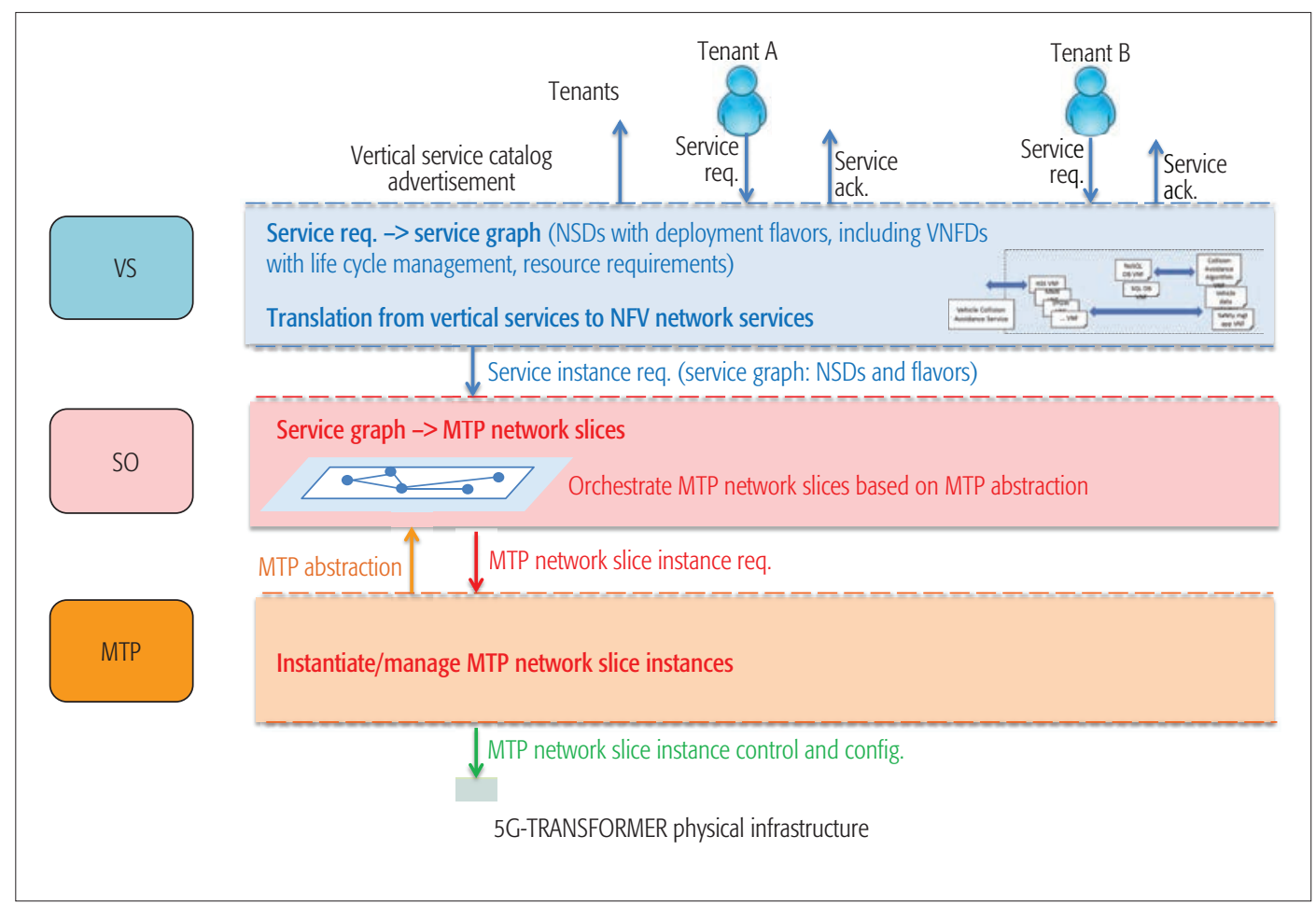

Figure 2. 5G-TRANSFORMER high level workflow for instantiation of a vertical service.

ments and functional description of the vertical slice to be created and deployed. The SO then interacts with other SOs to provide end-to-end network service delivery, through the SOSO-SAP interface.

The SO module next sends its instructions for resource allocation and monitoring to the underlying MTP module through the MTP-SAP interface. The MTP instantiates and controls the VNFs and MEC resources over physical substrates and optimizes their placement and migration. The MTP also exposes an abstract view of its context through analytics service to the SO for context-aware decisions by the SO.

\section{System Design AND Key R\&D Challenges SYSTEM DESIGN}

5G-TRANSFORMER proposes a novel design of the global system architecture, including the functions and procedures for creating and managing slices including MEC infrastructure. Beyond an evolved 4G/MEC architecture, 5G-TRANSFORMER proposes an open and flexible transport and computing platform at the "5G edge" tailored for verticals. It provides different levels of abstractions and ways for the composition and orchestration of services on individual network slices. Moreover, it leverages on the concept of network slicing and virtualization together with native SDN and NFV control to flexibly distribute VNFs in MEC and cloud platforms. Abstraction levels allow as a service resources provisioning for verticals' applications; slicing creation with required network functions and SLA; and processed/filtered monitoring features.

In order to build such an architecture, our idea is to extend the European Telecommunications Standards Institute (ETSI) management and orchestration (MANO) [1] base design adding new functional building blocks, namely the VS and SO interworking with the MTP.

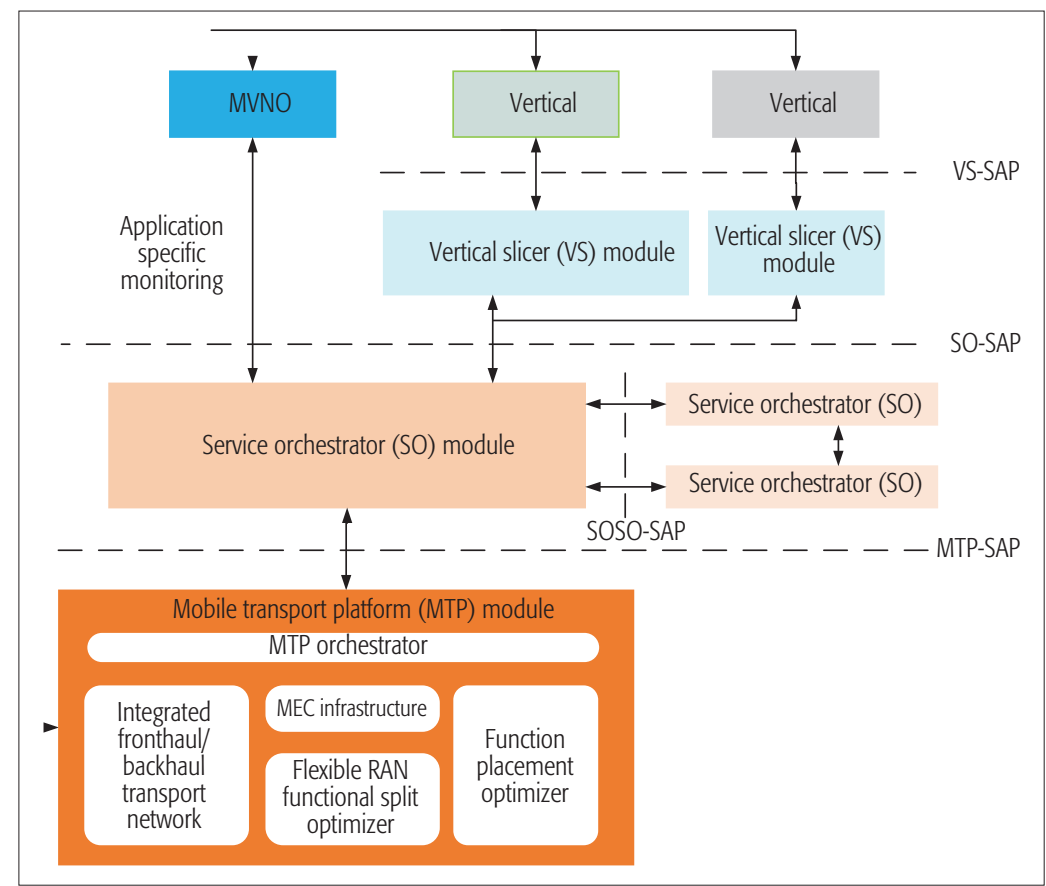

Figure 3. 5G-TRANSFORMER system view.

\section{VERTICAL SLICER}

The VS is a new component on top of the SO to create customized slices based on blueprints. Thereby, verticals can significantly reduce the time to create services.

This approach requires complex mechanisms for automatic service decomposition to translate a blueprint into a set of network graphs and requirements, algorithms for the dynamic and flexible placement of vertical functions, and abstract monitoring mechanisms to enable SLA verification to verticals. 


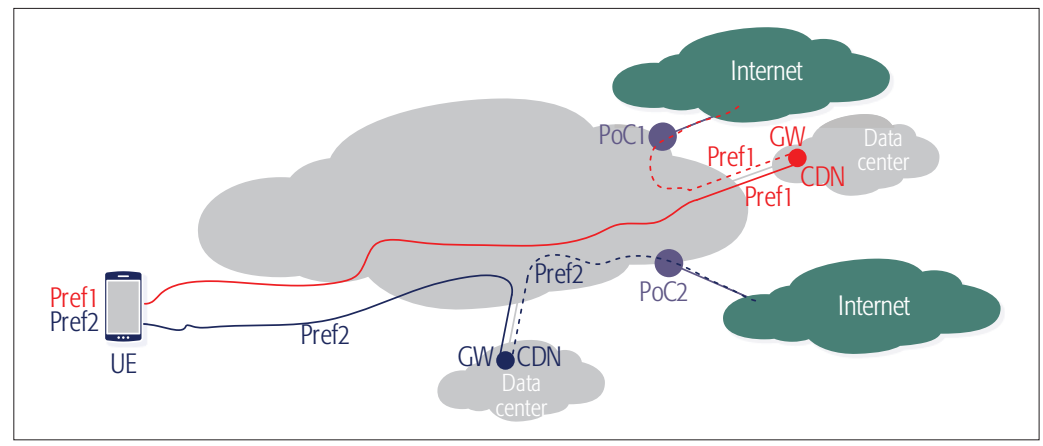

Figure 4. Emulated scenario. domains and manages their allocation to different MTP slices. The SO receives the service requirements via the SO-SAP interface from mobile (virtual) network operators (M[V]NOs) and/or vertical industries. The SO provides end-to-end network service delivery according to the network service requirement provided by the VS or MVNOs through deciding the optimal resource allocation and VNF/VA placement, and in turn instructing the configuration of resources of the local MTP and federated MTP domains.

SDN techniques are a valuable tool to support orchestration, especially in the case of highly mobile users. As a case in point for the sake of providing an intuitive validation of some of these concepts, we consider a sample network scenario (shown in Fig. 4) featuring an SDN-based backhaul network interconnecting points of access (PoAs) on the radio access network (RAN). A vertical requests a virtual content delivery network (CDN) service with enhanced mobility support. The CDN service is provided through VNFs deployed on compute nodes connected to the SDN-based backhaul. A distributed mobility management (DMM) service monitors the movements of the user equipments (UEs), and if a change of PoA is detected, it:

- Instantiates a new CDN node nearer to the new PoA.

- Reconfigures the SDN backhaul to route the flow to the new CDN node. In a legacy network, this procedure would be accomplished using Proxy Mobile IPv6 (PMIPv6) [11], a tunnel-based solution with well-known scalability issues.

We have emulated the scenario using a set of interconnected OpenFlow Ethernet switches with a WLAN interface working as PoA [12]. The forwarding is based on a direct modification of flow tables using standard OpenFlow rules. Results show that the SDN-based solution lowers the handover signaling cost compared to the PMIPv6 solution. Figure 5a highlights a minimal performance degradation as the number of SDN switches $(k)$ increases ( $\mu$ represents the mean of the exponential time between $\mathrm{MN}$ handovers). If PMIPv6 is used (Fig. 5b), the handover cost ramps up as handovers are more frequent, for different values of $\lambda$, the mean of the exponential interval that a tunnel remains active.

If the SO detects that one MTP domain alone does not have enough infrastructure resources to orchestrate the required service, it interacts with other SOs via the SOSO-SAP interface to compose service federation across multiple administrative domains. In this case, the SO will dynamically discover the available administrative domains by exchanging the view with the SOs of the neighboring domains, and negotiate with them to decide which administrative domains can be federated together to provide end-to-end service orchestration ensuring the desired SLAs.

Finally, to ensure that the service requirements are fulfilled, a flexible monitoring platform is required to collect and process consolidated monitoring data from multiple MTPs to monitor end-toend infrastructure services to support vertical service management at runtime. It will operate over multiple domains acting as a consumer of the monitoring services exposed by the MTPs. The platform will
The SO is in charge of end-to-end service orchestration and federation of transport networking and computing resources across one or multiple MTP 


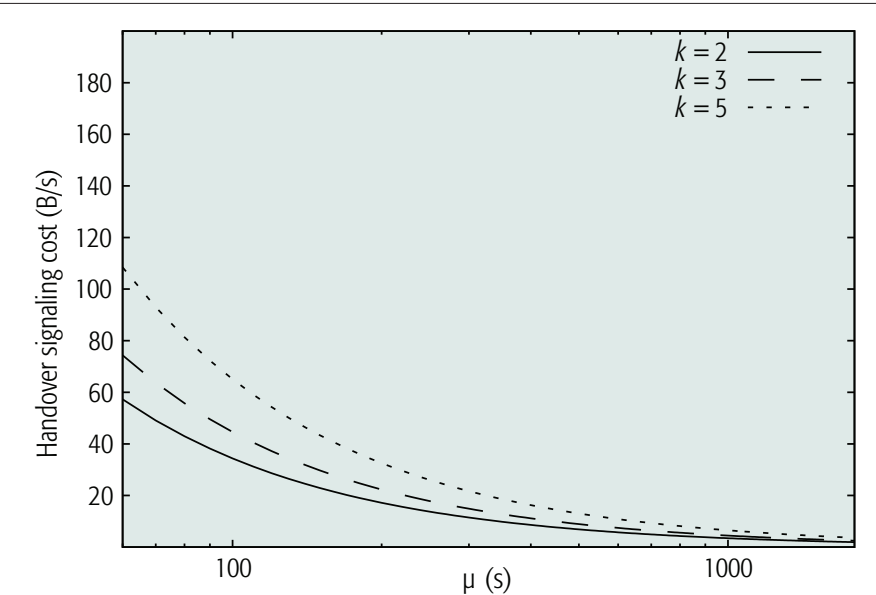

(a)

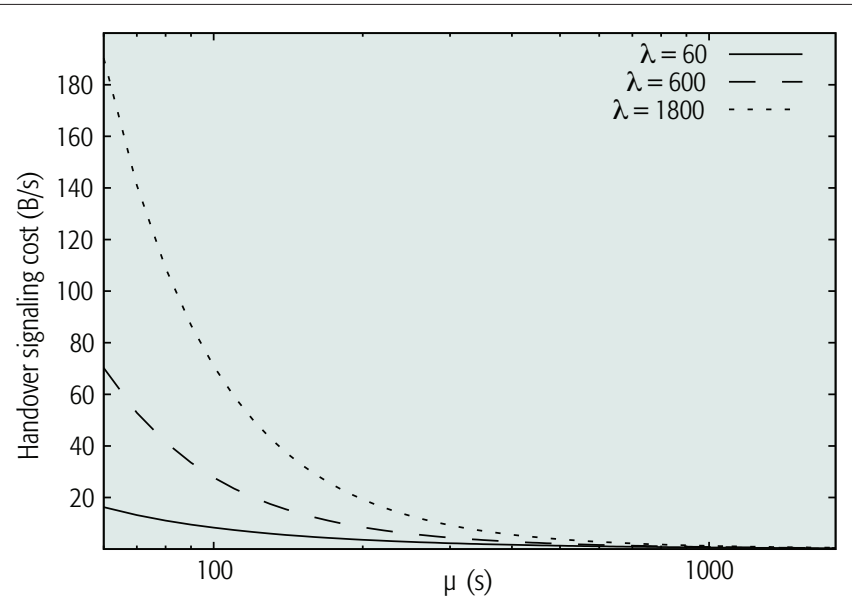

(b)

Figure 5. Handover signaling cost: a) SDN-based solution; b) PMIPv6-based solution.

offer a set of APIs for verticals to acquire monitoring data and expose it to the service part, integrating vertical-specific analytics algorithms.

In terms of possible implementation, the SO can be developed by extending existing open source MANO platforms (e.g., OSM, ${ }^{3}$ ONAP, 4 Cloudify ${ }^{5}$ ). The monitoring platform can be developed by extending existing open source monitoring tools (e.g., Prometheus, ${ }^{6} \mathrm{Zabbix}^{7}$ ) and integrating further components for efficient storage and access of distributed data (e.g., Cassandra databases).

\section{MOBILE TRANSPORT PLATFORM}

The MTP consists of the actual infrastructure (physical or virtual) over which vertical slices are created. The MTP solution is based on the ETSI NFV MANO to support vertical services by:

- Receiving service requirements including MEC service parameters (e.g., amount of processing, bandwidth, latency of the services, resource availability, and hardware characteristics) from the SO

- Identifying the geographical location of servers working on a suitable abstraction view that allows cross-optimization of mobile, transport, and computing

- Triggering the virtual infrastructure manager (VIM) for configuration of servers in data centers having radio and transport resources for connectivity

- Configuring the virtual function (VF) and, when needed, also the radio and transport resources by triggering the corresponding controllers

In the following lines, we present the details on the operation of the MTP and its relation with the VS and the SO. The MTP exposes to the SO a suitable abstract view of the processing and storage available resources, allowing the SO to select them according to the requirements received from the VS. The abstract view exposed to the SO by the MTP includes not only the processing and storage resources allocated to the vertical, but also the virtual links used to connect those resources. With this abstract view, the MTP is able to translate it to the corresponding requirements for the radio/mobile and transport composing the selected virtual links. In a second step, the
MTP selects and configures the related physical resources. Given that the chain of the nodes composing the mobile communication (e.g., the distributed unit and the centralized unit in a functional split) are connected by transport links/ networks, the selection of the current physical resources within the MTP is based on an implementation-dependent cross-optimization among mobile and transport resources.

The presented 5G-TRANSFORMER architecture allows the vertical to ignore the detailed requirements of the infrastructure that provides the services (i.e., the mobile-transport infrastructure), while it simplifies the operations on the mobile and transport infrastructure that can be operated as separate layers interacting in a sort of client-server relationship.

\section{SUMMARY AND OUTLOOK}

5G systems give rise to a wide range of vertical industries with very diverse and stringent service requirements. To enable this vision, the 5G-TRANSFORMER approach proposed in this article is to blend together SDN, NFV, and MEC technologies to create logical infrastructures for meeting the networking and computing requirements of vertical industries, particularly the ones requiring low-latency such as automotive, eHealth, and media. The 5G-TRANSFORMER solution therefore builds on three pillars:

1. Virtualization of the mobile transport network infrastructure

2. Network slicing enabling per-slice management of the virtualized resources

3. Integration of MEC to enable the deployment of low-latency services and VNFs at the edge.

The 5G-TRANSFORMER solution combines three novel building blocks:

- The vertical slicer as the logical entry point for verticals to support easy creation and management of the slices

- The service orchestrator for end-to-end service orchestration, federation of transport networking and computing resources from multiple domains, and their allocation to slices

- MTP as the underlying unified transport stratum for integrated fronthaul and backhaul networks

\footnotetext{
3 Open Source MANO, https://osm.etsi.org/

4 ONAP, https://www.onap. org/

${ }^{5}$ Cloudify, https://cloudify. $\mathrm{CO} /$

6 Prometheus, https://prometheus.io/

7 Zabbix, https://www.zabbix.com/
} 
5G systems gives rise to

a wide range of vertical industries with very

diverse and stringent

service requirements.

To enable this vision,

the 5G-TRANSFORMER

approach proposed in

this article is to blend

together SDN, NFV and

MEC technologies to

create logical infrastruc-

tures for meeting the

networking and com-

puting requirements of

vertical industries.
The design of these three building blocks together with the global system architecture are the main research challenges tackled. The targeted solution includes:

- Procedures for creating and managing slices including MEC infrastructure

- The definition of vertical service descriptors based on blueprints and an easy-to-use interface for verticals to deploy them on the slices of the underlying platform

- The design of network service descriptors in the SO and its interfaces to the VS, MTP, and other SOs

- Novel service orchestration and federation algorithms to optimize resource allocation across one or multiple MTP domains, tailored for different slices

- Focusing on transport abstractions supporting efficient functional splits of virtualized radio software stacks

\section{ACKNOWLEDGMENTS}

This work has been partially supported by the EU H2020 5GPPP 5G-TRANSFORMER project (Grant 761536).

\section{REFERENCES}

[1] ETSI, Network Functions Virtualisation, "Network Functions Virtualisation (NFV); Management and Orchestration," Dec. 2014

[2] R. Mijumbi et al., "Network Function Virtualization: State-ofthe-Art and Research Challenges," IEEE Commun. Surveys \& Tutorials, vol. 18, no. 1, 2016, pp. 236-62.

[3] N. Alliance, "Description of Network Slicing Concept," NGMN 5G P, vol. 1, 2016.

[4] Y. C. Hu et al., "Mobile Edge Computing - A Key Technology Towards 5G," ETSI White Paper, vol. 11, 2015.

[5] X. Costa-Pérez et al., "5G-Crosshaul: An SDN/NFV Integrated Fronthaul/Backhaul Transport Network Architecture\}," IEEE Wireless Commun., vol. 24, no. 1, Feb. 2017, pp. 38-45.

[6] A. Ksentini and N. Nikaein, "Toward Enforcing Network Slicing on RAN: Flexibility and Resources Abstraction," IEEE Commun. Mag., vol. 55, no. 6, June 2017, pp. 102-08.

[7] X. Li et al.," $5 \mathrm{G}-\mathrm{Crosshaul} \mathrm{Network} \mathrm{Slicing:} \mathrm{Enabling}$ Multi-Tenancy in Mobile Transport Networks," IEEE Commun. Mag., vol. 55, no. 8, Aug. 2017, pp. 128-37.

[8] J. O. Fajardo et al., "Introducing Mobile Edge Computing Capabilities Through Distributed 5G Cloud Enabled Smal Cells," Mobile Networks and Applications, vol. 21, no. 4, 2016, pp. 564-74.

[9] C. J. Bernardos et al., "5GEx: Realising A Europe-Wide Multi-Domain Framework for Software-Defined Infrastructures," Trans. Emerging Telecommun. Technologies, vol. 27, no. 9, 2016, pp. 1271-80.

[10] R. Muñoz et al., "The Need for a Transport API in 5G Networks: The Control Orchestration Protocol," OFC 2016 2016, pp. 1-3.

[11] S. Gundavelli et al., "Proxy Mobile IPv6," IETF RFC 5213, Aug. 2008.

[12] L. Cominardi et al., "Distributed Mobility Management Solutions for Next Mobile Network Architectures," Computer Networks, vol. 121, 5 July 2017, pp. 124-136.

\section{BIOGRAPHIES}

ANTONIO DE LA OLIVA received his telecommunications engineering degree in 2004 and his Ph.D. in 2008 from the Universidad Carlos III Madrid (UC3M), Spain, where he has been an associate professor since then. $\mathrm{He}$ is an active contributor to IEEE 802 where he has served as Vice-Chair of IEEE 802.21b and Technical Editor of IEEE 802.21d. He has also served as a Guest Editor of IEEE Communications Magazine. He has published more than 30 papers on different networking areas.

$\mathrm{XI} \mathrm{LI}$ is a senior researcher in 5G Networks R\&D at NEC Laboratories Europe $\mathrm{GmbH}$. She has actively participated in the EU H2020 5GPPP projects (5G-Transformer and 5G-Crosshaul) and contributed to standardization (IETF, ONF) on microwave/ mmWave modeling. Previously, she was a researcher and lecturer at the University of Bremen and a solution designer at
Telefonica. She received her M.Sc. in 2002 from the Technica University of Dresden and her Ph.D. in 2009 from the University of Bremen.

XAVIER COSTA-PÉrEZ is head of 5G Networks R\&D and deputy general manager of the Security \& Networking R\&D Division at NEC Laboratories Europe. His team contributes to products' roadmap evolution as well as to European Commission R\&D collaborative projects, and has received several awards for successful technology transfers. He received both his M.Sc. and Ph.D degrees in telecommunications from Universidad Politécnica de Catalunya (UPC) and was the recipient of a national award for his Ph.D. thesis.

CARLOS J. BERNARDOS received a telecommunication engineering degree in 2003 and a Ph.D. in telematics in 2006, both from UC3M where he has been an associate professor since 2008. His current work focuses on virtualization in heterogeneous wireless networks. He has published over 70 scientific papers in international journals and conferences, and he is an active contributor to the IETF. He has served as a Guest Editor of IEEE Network.

PHILIPPE BERTIN is a senior research engineer at Orange Labs $\mathrm{He}$ is managing future networks research projects with $\mathrm{b}<>\mathrm{com}$ Institute for Research and Technology. His research addresses the design of distributed and dynamic control and data planes for flexible and convergent 5G networks leveraging on software defined networking and network virtualization. He is co-author of 50+ publications and 11 patents. He graduated from Paris 6 University (M.Sc.) and Rennes University (Ph.D.).

PaOla IOVAnNa is a master researcher at Ericsson Research. She has experience in packet over optical networking, with special focus on traffic routing, transport network control, and related technologies such as IP/MPLS/Ethernet, WDM, and SDN. She leads a research team defining networking and control solutions for $5 \mathrm{G}$ transport. She has 20 years of experience in this area, and is the author of more than 70 patents and numerous publications.

THOMAS DEISS received his degree in computer science in 1990 and his Ph.D. in 1999 from the University of Kaiserslautern. He joined Nokia in 1999. He has contributed to standardization on automated testing and worked in requirements engineering for backhaul functionality of WCDMA and LTE base stations with a focus on backhaul sharing among radio technologies. He participated in the $\mathrm{H} 2020$ phase 1 project 5G-Crosshaul.

JOSEP MANGUES is a senior researcher and head of the Communication Networks Division of the CTTC. He has participated in several publically funded and industrial research projects (e.g. 5GPPP 5G-Transformer and 5G-Crosshaul). He is Vice-Chair of IEEE WCNC 2018, Barcelona, Spain. Previously, he was also a researcher and assistant professor at UPC, from which he received his degree and Ph.D. in telecommunications in 1996 and 2003, respectively. His research interests are SDN, NFV, and MEC.

ALAIN MOURAD is leading the research and development of next generation radio access networks at InterDigital Internationa Labs. Prior to joining InterDigital, he was a principal engineer at Samsung Electronics R\&D, United Kingdom, and a senior engineer at Mitsubishi Electric R\&D Centre Europe, France. Throughout his career, he has been active in the research and standardization of recent communication networks (5G, 4G $3 \mathrm{G})$ and broadcasting systems (ATSC 3.0 and DVB-T2/NGH).

Claudio Casetti [M'05, SM'17] received his Ph.D. degree in electronic engineering in 1997 from Politecnico di Torino, where he is currently an associate professor. He has coauthored almost 200 papers in the fields of transport protocols, mobile networks, and SDN networks. He holds three patents.

JOSE ENRIQUE GONZALEZ is a senior project manager in Atos Spain's Research and Innovation Department. He holds a degree in telecommunications engineering and a Master's in business administration from the Universidad Politécnica de Madrid (UPM) and a Master's in human resources and executive and business coaching from the Instituto Europeo de Estudios Empresariales. $\mathrm{He}$ is currently working on several EC R\&D projects.

ARTURO AZCORRA received his M. Sc. degree in telecommunications engineering from UPM in 1986 and his Ph.D. in 1989 In 1993, he obtained an M.B.A. with honors from Instituto de Empresa. He has participated in more than 50 research projects. He has coordinated the CONTENT and E-NEXT European Networks of Excellence, and the CARMEN, 5G-Crosshaul, and $5 \mathrm{G}$-TRANSFORMER EU projects. He is the founder and director of the international research center IMDEA Networks. 